\title{
Energy handling in renal tubular epithelial cells of the hamster, a native hibernator, under warm anoxia or reoxygenation
}

\author{
THEODOROS ELEFTHERIADIS, GEORGIOS PISSAS, GEORGIA ANTONIADI, SPYRIDON GOLFINOPOULOS, \\ VASSILIOS LIAKOPOULOS and IOANNIS STEFANIDIS
}

Department of Nephrology, Faculty of Medicine, University of Thessaly, 41110 Larissa, Greece

Received August 24, 2018; Accepted October 10, 2018

DOI: $10.3892 /$ br.2018.1157

\begin{abstract}
Ischemia-reperfusion (I-R) injury causes several diseases, including acute kidney injury. Hibernating mammals survive periods of torpor with a marked drop in tissue perfusion, interspersed with periods of arousal, and consequently I-R injury. In the present study, sensitivity to anoxia and/or reoxygenation and alterations in cellular ATP and homeostasis of the two most energy consuming processes, protein translation and $\mathrm{Na}^{+}-\mathrm{K}^{+}$-ATPase function, were evaluated in renal proximal tubular epithelial cells of mouse or native hibernator hamster origin. Compared with the mouse cells, the hamster cells were less sensitive to anoxia and reoxygenation and ATP was preserved under anoxia. Anoxia triggered mechanisms that suppress protein translation in both species. However, under anoxia, the activity of ATPase, which is mostly attributed to $\mathrm{Na}^{+}-\mathrm{K}^{+}$-ATPase function, remained stable in the hamster cells but decreased in the mouse cells. In normoxia, ATPase activity in hamster cells was considerably lower than that in mouse cells. As the $\mathrm{Na}^{+}-\mathrm{K}^{+}$-ATPase pump preserves the ion gradient against passive leakage through ion channels, the lower energy demand for the function of this pump in hamster cells may indicate less ion leakage due to fewer ion channels. In accordance with this hypothesis, ouabain-treated hamster cells had a higher survival rate than mouse cells, indicating fewer ion channels and consequently slower deregulation of intracellular ion concentration and cell death due to $\mathrm{Na}^{+}-\mathrm{K}^{+}$-ATPase inhibition. Therefore, it is likely that the conserved energy from the suppression of protein translation is adequate enough to support the lower energy demand for $\mathrm{Na}^{+}-\mathrm{K}^{+}$-ATPase function and cell survival of hamster cells under anoxia. Clarifying how cells of a native hibernator manage energy under warm I-R may reveal novel and possible clinically applicable pathways for preventing I-R injury.
\end{abstract}

Correspondence to: Professor Theodoros Eleftheriadis, Department of Nephrology, Faculty of Medicine, University of Thessaly, Mezourlo Hill, 41110 Larissa, Greece

E-mail: teleftheriadis@yahoo.com

Key words: ischemia-reperfusion, hibernation, kidney, ATP, protein translation, $\mathrm{Na}^{+}-\mathrm{K}^{+}$-ATPase

\section{Introduction}

Ischemia-reperfusion (I-R) injury is the consequence of anoxia due to ischemia of a tissue resulting from an obstructed artery or circulation collapse and the marked production of reactive oxygen species following re-canalization of the blocked artery or restoration of effective blood volume. Therefore, I-R injury is important in the pathogenesis of several human diseases, including coronary heart disease, cerebral ischemia and multiple organ failure (1-3). The kidney is particularly prone to I-R injury as the partial pressure of oxygen is relatively low in this organ and renal tubular epithelial cells require large amounts of energy to preserve water, acid-base and electrolyte homeostasis $(4,5)$.

Several mammals hibernate during the winter months in order to cope with a scarcity of food. Hibernation is characterized by prolonged periods of deep torpor with a rapid fall in body temperature, heart rate and breathing, with the whole organism being in an ischemic state. Deep torpor is interrupted by short periods of arousal when the animals rewarm themselves back to euthermia and restore heart rate and breathing for several hours, setting the organism in a state of reperfusion. Intriguingly, these animals survive without signs of I-R injury in the brain, heart, kidneys or other organs $(6,7)$.

Classically, mammalian hibernation is considered to represent a state of resistance to cold I-R injury, although body temperature is restored during interbout arousals $(6,7)$. However, studies have demonstrated that these mammals resist warm I-R injury more than other phylogenetically related species that are unable to hibernate $(8,9)$. Evidence has revealed hibernation in high ambient and body temperatures, a phenomenon observed even in primates $(10,11)$. Therefore, besides resistance to cold I-R injury, resistance to warm I-R injury also occurs in certain hibernators, some of which are phylogenetically close to humans. These data indicate that, under certain circumstances, human cells may also be able to become resistant to warm I-R injury, making the investigation of this phenomenon interesting from a clinical point of view.

One of the events that requires further investigation is the preservation of energy homeostasis in hibernators during I-R. For this purpose, the present study compared the effects of warm I-R on two of the most energy demanding cellular processes, protein translation and the activity of the $\mathrm{Na}^{+}-\mathrm{K}^{+}$-ATPase pump. Of the $80 \%$ of oxygen consumption 
coupled to ATP synthesis, $25-30 \%$ is used for protein synthesis and $19-28 \%$ is used by $\mathrm{Na}^{+}-\mathrm{K}^{+}$-ATPase. Experiments in rats have shown that the percentage of ATP consumed for the $\mathrm{Na}^{+}-\mathrm{K}^{+}$-ATPase function in the mammalian kidney tissue is even higher (12).

In the present study, primary renal proximal tubular epithelial cells (RPTECs), which are sensitive to hypoxia $(4,5)$, of mouse or native hibernator Syrian hamster origin were cultured at $37^{\circ} \mathrm{C}$ under normoxia, anoxia or anoxia followed by reoxygenation. Investigating the mechanisms that offer mammalian hibernators resistance to I-R injury may reveal novel therapeutic strategies for attenuating I-R injury in humans.

\section{Materials and methods}

Cell culture conditions. Primary Syrian hamster RPTECs (cat. no. HM-6015) and primary C57BL/6 Mouse RPTECs (cat. no. C57-6015) were cultured with the Complete Epithelial Cell Medium/w kit (cat. no. M6621) (all from Cell Biologics, Chicago, IL, USA), supplemented with epithelial cell growth supplement (insulin-transferrin-selenium and epidermal growth factor), antibiotic-antimycotic solution (penicillin, streptomycin and amphotericin B) and 2\% fetal bovine serum (all from Cell Biologics). The cells were seeded in 6-well plates at a density of 300,000 cells per well, in 12-well plates at a density of 100,000 cells per well or in 96-well plates at a density of 10,000 cells per well for $16 \mathrm{~h}$ prior to the onset of anoxic conditions. The GasPak ${ }^{\mathrm{TM}}$ EZ Anaerobe Container System with Indicator (cat. no. 26001; BD Biosciences, Franklin Lakes, NJ, USA) was used to reduce oxygen levels $<1 \%$. Cells within the anaerobe container were cultured at $37^{\circ} \mathrm{C}$. These anoxic conditions imitate warm ischemia. The reoxygenation experiments started following $24 \mathrm{~h}$ of anoxia. In the experimental group exposed to reoxygenation, the cells were washed with Dulbecco's phosphate buffer saline (Sigma-Aldrich; Merck KGaA, Darmstadt, Germany), fresh supplemented culture medium was added, and the cells were incubated at $37^{\circ} \mathrm{C}$ in a humidified atmosphere containing $5 \% \mathrm{CO}_{2}$. These reoxygenation conditions imitate warm reperfusion. Whenever cell manipulation under anoxic conditions was necessary, this was performed within an anoxic chamber. All the experiments were performed nine times.

Evaluation of cell death, cellular ATP content and ATPase activity. Cell imaging was performed to assess the sensitivity of mouse and hamster RPTECs to warm anoxia or reoxygenation in cells cultured in 12-well plates. For this purpose, an inverted microscope (Axiovert 40C; Carl Zeiss AG, Göttingen, Germany) and a digital camera with the related software (3MP USB2.0; Microscope Digital Camera, Amscope, Irvine, CA, USA) were used. The cells under anoxic conditions were monitored within their anoxic container, which was transparent permitting live image capture. As cell staining was not possible in living cells within the anoxic container, cell death was also evaluated biochemically. Cell death was assessed using the Cell Death Detection ELISA Plus kit (cat. no. 11774425001 ver. 11; Roche Diagnostics, Indianapolis, IN, USA), which relies on the detection of cytoplasmic histone-associated DNA fragments. The RPTECs were cultured in 6-well plates for $24 \mathrm{~h}$ in anoxia, and for another $2 \mathrm{~h}$ they were reoxygenated in the case of the reoxygenation procedure. The respective time points were selected according to the results obtained by cell imaging.

Cellular ATP content was assessed using the ATP Colorimetric/Fluorometric Assay kit (cat. no. MAK190, Sigma-Aldrich; Merck KGaA) in RPTECs cultured in 96-well plates.

Cellular ATPase activity was assessed using the ATPase Activity Assay kit (colorimetric; cat. no. K417-100; BioVision, Inc., Milpitas, CA, USA) in RPTECs cultured in 6-well plates. The principle of this assay lies in the detection of a stable chromophore generated by the release of a free phosphate ion derived from ATP hydrolysis. The activity is expressed as free phosphate production as $\mathrm{nmol} / \mathrm{min}$ (or $\mathrm{mU}) / 100 \mu \mathrm{g}$ of cellular protein.

Evaluation of proteins that control protein translation and activated AMP-activated protein kinase (AMPK). The cells were cultured in 6-well plates as described above. The cells were then lysed using T-PER tissue protein extraction reagent (Thermo Fisher Scientific, Inc., Waltham, MA, USA) supplemented with protease and phosphatase inhibitors (Sigma-Aldrich; Merck KGaA and Roche Diagnostics, respectively). Protein was quantified using a Bradford assay (Sigma-Aldrich; Merck KGaA) and $10 \mu \mathrm{g}$ from each sample was used for western blotting. Protein samples were electrophoresed in a 4-12\% bis-tris acrylamide gel (NuPAGE 4-12\% Bis-Tris Gel $1.0 \mathrm{~mm}$ x 15 well; cat. no. NP0323BOX, Invitrogen; Thermo Fisher Scientific, Inc.) at $180 \mathrm{~V}$ constant for $30 \mathrm{~min}$. Blotting of the electrophoresed gel proteins on the polyvinylidene difluoride (PVDF) membrane was performed via electroporation at $30 \mathrm{~V}$ constant for $1 \mathrm{~h}$. Skimmed milk in tris-buffered saline with Tween-20 was used for blocking. The blots were incubated with primary antibody against phosphorylated Ser51 eukaryotic translation initiation factor $2 \alpha$ (p-eIF $2 \alpha$; dilution 1:500; cat. no. 9721), phosphorylated Thr37/46 eukaryotic translation initiation factor 4E-binding protein 1 (p-4E-BP1; dilution 1:1,000; cat. no. 2855), phosphorylated Thr172 $\alpha$ subunit of AMPK (p-AMPK; dilution 1:1,000; cat. no. 2535) and $\beta$-actin (dilution 1:2000; cat. no. 4967) (all from Cell Signaling Technology, Inc., Danvers, MA, USA) for $16 \mathrm{~h}$ at $4^{\circ} \mathrm{C}$. This was followed by $30 \mathrm{~min}$ of incubation at room temperature with the secondary antibody (anti-rabbit IgG, HRP-linked; dilution 1:1,000; cat. no. 7074, Cell Signaling Technology, Inc.). In reprobing the PVDF blots, the previous primary and secondary antibodies were removed with Restore Western Blot Stripping Buffer (Thermo Fisher Scientific, Inc.). Densitometric analysis of the western blot bands was performed using ImageJ software (National Institute of Health, Bethesda, MD, USA).

Evaluation of resistance to ouabain-induced cell death. The resistance of the mouse or hamster RPTECs to ouabain-induced cell death was assessed by cell imaging in cells cultured in 12-well plates under normoxic conditions $\left(37^{\circ} \mathrm{C}\right.$ in a humidified atmosphere containing $\left.5 \% \mathrm{CO}_{2}\right)$ with or without the presence of ouabain (cat. no. O3125, Sigma-Aldrich; Merck KGaA) at a concentration of $2 \mathrm{mM}$. Ouabain is a well-known $\mathrm{Na}^{+}-\mathrm{K}^{+}$-ATPase inhibitor. It exerts its effect mainly by disrupting the ion gradient of the cell. However, evidence supports that $\mathrm{Na}^{+}-\mathrm{K}^{+}$-ATPase may also be a receptor and signal transducer, and that its stabilization by ouabain exerts additional, but less profound effects (13). 
A

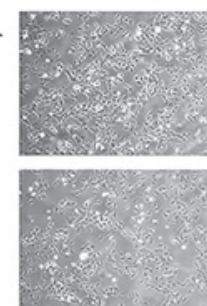

Anox 0h
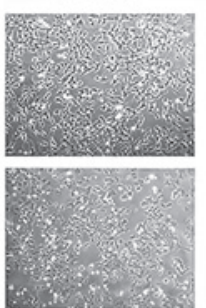

Anox 24h

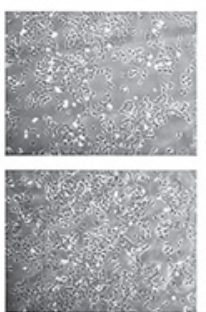

Anox $12 \mathrm{~h}$

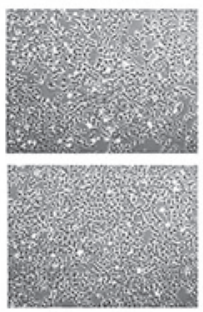

Re-ox 2h

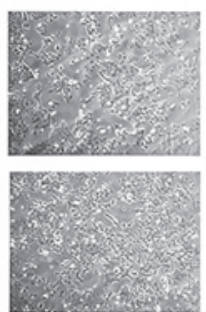

Anox 24h

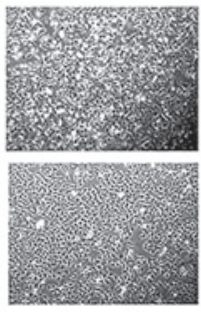

Re-ox 4h
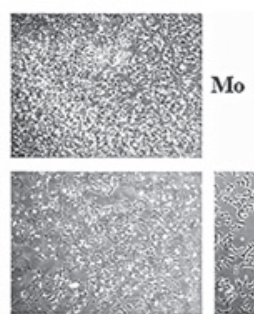

Anox 48h

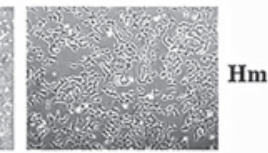

Anox 96h
B

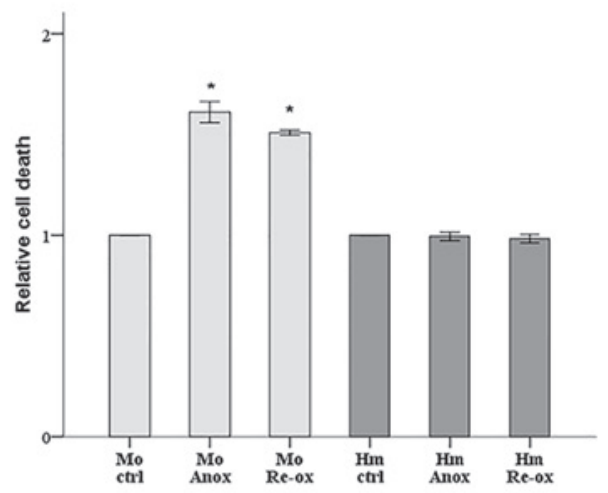

Figure 1. Cell death due to warm anoxia or reoxygenation in mouse and hamster renal proximal tubular epithelial cells. (A) Cell imaging (magnification, $\mathrm{x} 100)$ and (B) cell death assay revealed that, in contrast to mouse cells, hamster cells were resistant to cell death caused by warm anoxia or reoxygenation. All experiments were performed nine times. ${ }^{*} \mathrm{P}<0.05$ vs. ctrl. Error bars correspond to the mean \pm standard deviation. Mo, mouse; Hm, hamster; Anox, anoxia; ctrl, control culture under normoxia; Re-ox, reoxygenation.

Various concentrations of ouabain have been used in several studies showing a varying degree of $\mathrm{Na}^{+}-\mathrm{K}^{+}$-ATPase inhibition (14-16). In the present study, the high concentration of $2 \mathrm{mM}$ was selected to achieve the highest possible inhibition of $\mathrm{Na}^{+}-\mathrm{K}^{+}$-ATPase. For this purpose, images were captured hourly using an inverted microscope (Axiovert 40C; Carl Zeiss AG) and a digital camera with the related software (3MP USB2.0, Microscope Digital Camera; AmScope, Irvine, CA, USA).

In addition to cell imaging, cell survival was assessed biochemically in cultures of RPTECs in 96-well plates under normoxic conditions, with or without the presence of $2 \mathrm{mM}$ ouabain, for $4 \mathrm{~h}$. This time point was selected according to the results obtained by cell imaging. For this purpose, the TACS XTT assay kit (cat. no. 4891025K; Trevigen, Inc., Gaithersburg, MD, USA) was used according to the protocol provided by the manufacturer.

Statistical analysis. The normality of the evaluated variables was assessed and confirmed using a one-sample Kolmogorov-Smirnov test. For comparison of means, an unpaired t-test or one-way analysis of variance followed by Bonferroni's correction test was used. For statistical analysis of the cell imaging results, the Mann-Whitney U test was used as these results did not fit the normal distribution. The results are expressed as the mean \pm standard deviation and a $\mathrm{P}<0.05$ was considered to indicate a statistically significant difference. Although the original data were analyzed statistically, for reader's convenience, in the majority of cases, the results are shown following normalization of means for the control group. Statistical analysis was performed with IBM SPSS Statistics for Windows, version 20 (IBM Corp., Armonk, NY, USA).

\section{Results}

In contrast to mouse cells, hamster cells are resistant to cell death due to warm anoxia or reoxygenation. Cell imaging revealed that the mouse RPTECs died after $48 \mathrm{~h}$ under anoxic conditions, whereas the hamster RPTECs remained viable even after $96 \mathrm{~h}$. The mouse RPTECs were also more sensitive to reoxygenation, as their morphology deteriorated significantly after $4 \mathrm{~h}$, exhibiting condensation and loss of adherence on a large scale, whereas the hamster cells exhibited none of these signs after $12 \mathrm{~h}$ (Fig. 1A).

The cell imaging results were also confirmed biochemically. The hamster RPTECs resisted $24 \mathrm{~h}$ of warm anoxia and $2 \mathrm{~h}$ of reoxygenation. By contrast, the cell death assay revealed that mouse RPTECs were susceptible to both deleterious conditions, as cell death increased by $61 \%$ during anoxia and $51 \%$ during reoxygenation (Fig. 1B).

In mouse cells, cellular ATP content decreases under warm anoxia or reoxygenation but remains constant in hamster cells under anoxia. Compared with the control, cellular ATP content in the mouse RPTECs decreased by $38 \%$ following $24 \mathrm{~h}$ of anoxia and remained decreased, by $41 \%$, following $2 \mathrm{~h}$ of reoxygenation. In the hamster RPTECs, cellular ATP content did not alter significantly under anoxia but decreased by $39 \%$ following reoxygenation (Fig. $2 \mathrm{~A}$ ).

The levels of activated p-AMPK also confirmed the ATP content results as this enzyme is a sensor and regulator of cellular energy. In the mouse RPTECs, anoxia and reoxygenation increased the level of p-AMPK to 244 and $242 \%$ of the control level, respectively. In the hamster RPTECs, p-AMPK remained stable under anoxia but increased to $241 \%$ of the control level following reoxygenation (Fig. 2B and C).

Activated regulators of the protein translation eIF $2 \alpha$ and $4 E-B P 1$ follow the same trend in mouse and hamster cells under warm anoxia, but are overcorrected in hamster cells following reoxygenation. In the mouse RPTECs, the level of p-eIF $2 \alpha$ increased by $56 \%$ following anoxia and returned to the level of the normoxic conditions following reoxygenation. In the hamster RPTECs, anoxia increased the level of p-eIF2 $\alpha$ to $249 \%$ of the control, whereas reoxygenation decreased p-eIF $2 \alpha$ by $42 \%$ (Fig. 3A and B). 


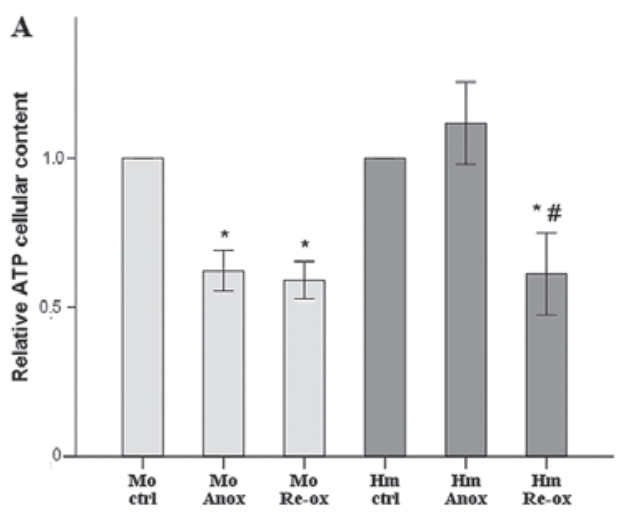

B

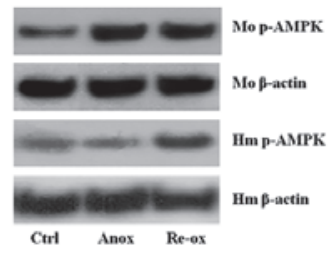

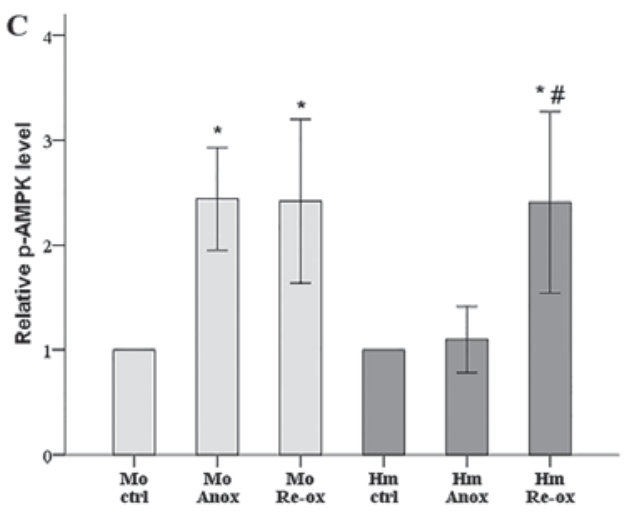

Figure 2. Cellular ATP content following warm anoxia or reoxygenation in mouse and hamster renal proximal tubular epithelial cells. (A) ATP assay revealed that, in mouse cells, cellular ATP content decreased under warm anoxia or reoxygenation. In hamster cells, ATP remained constant under anoxia and decreased following reoxygenation. (B) To confirm the results of the ATP assay, western blotting was performed to assess the activation status of the cellular sensor of energy AMPK; results of one of the nine performed experiments are presented. (C) Alterations in the levels of activated p-AMPK were in an opposite direction to the alterations of cellular ATP content. All experiments were performed nine times. ${ }^{*} \mathrm{P}<0.05$ vs. ctrl; ${ }^{\sharp} \mathrm{P}<0.05$ vs. Anox. Error bars correspond to the mean \pm standard deviation. Mo, mouse; Hm, hamster; Anox, anoxia; ctrl, control culture under normoxia; Re-ox, reoxygenation; AMPK, AMP-activated protein kinase; p-AMPK, phosphorylated AMPK.

A

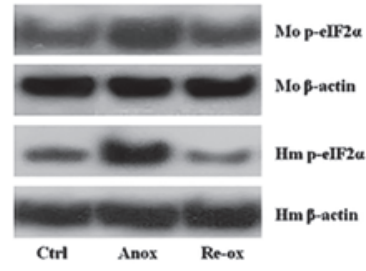

C

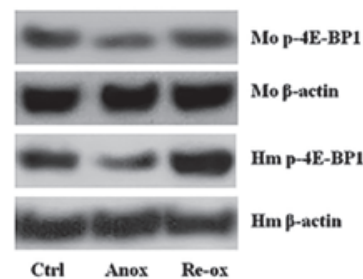

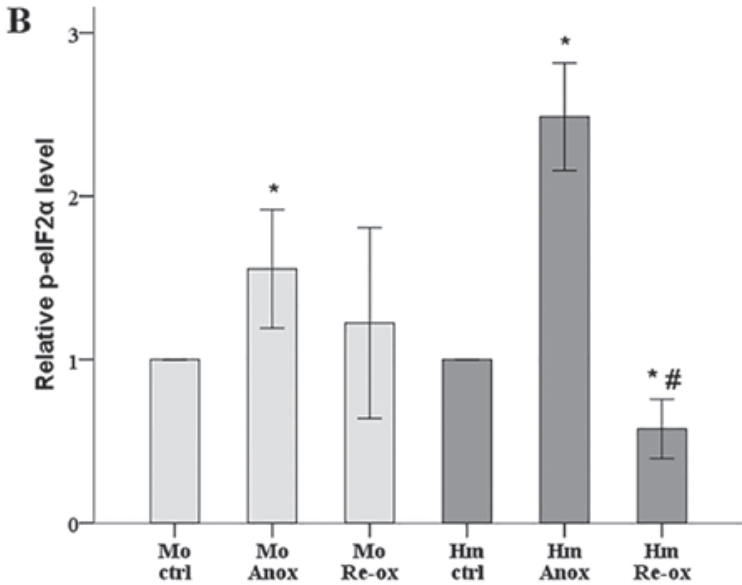

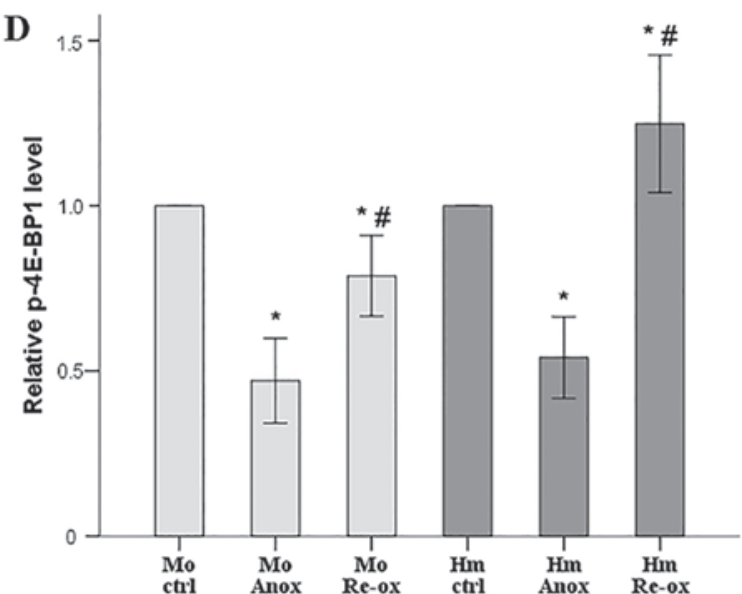

Figure 3. Activation status of protein translation regulators eIF2 $\alpha$ and 4E-BP1 following warm anoxia or reoxygenation in mouse and hamster RPTECs (A) Western blot analysis was performed to assess the level of p-eIF2 $\alpha$, and the results of one of the nine experiments are shown. (B) In mouse RPTECs, the level of p-eIF2 $\alpha$ increased following anoxia and returned towards the level measured in normoxic conditions following reoxygenation. In hamster RPTECs, p-eIF2 $\alpha$ increased following anoxia but overcorrected and was decreased following reoxygenation. (C) Levels of p-4E-BP1 from one of the nine performed experiments are shown. (D) In mouse RPTECs, the level of p-4E-BP1 decreased following anoxia and increases following reoxygenation, but remained lower compared with the level in normoxic conditions. In hamster RPTECs, the level of p-4E-BP1 decreased following anoxia but overcorrected and increased following reoxygenation. All experiments were performed nine times. ${ }^{*} \mathrm{P}<0.05$ vs. ctrl; " $\mathrm{P}<0.05$ vs. Anox. Error bars correspond to the mean \pm standard deviation. RPTECs, renal proximal tubular epithelial cells; Mo, mouse; Hm, hamster; Anox, anoxia; ctrl, control culture under normoxia; Re-ox, reoxygenation; p-eIF2 $\alpha$, phosphorylated eukaryotic translation initiation factor $2 \alpha$; p-4E-BP1, phosphorylated eukaryotic translation initiation factor 4E-binding protein 1. 


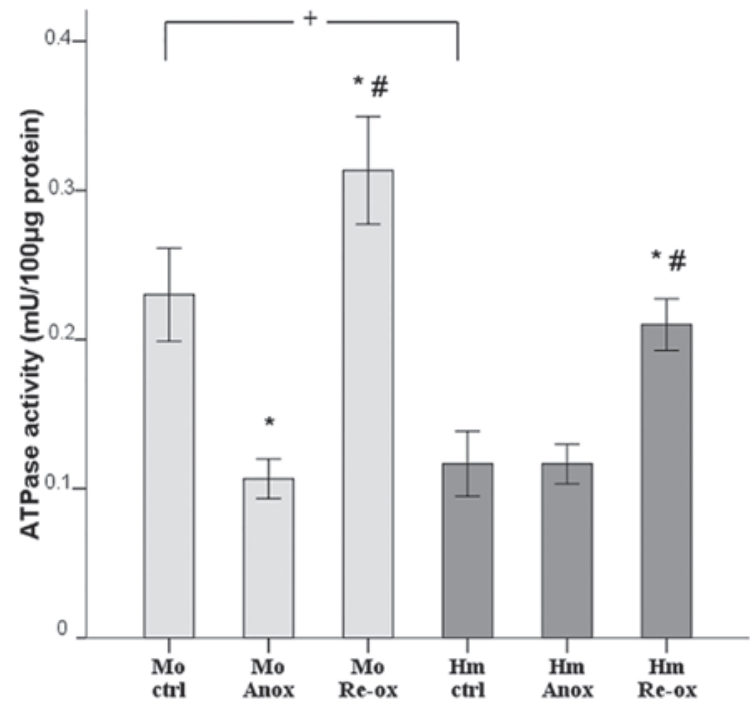

Figure 4. ATPase activity under warm anoxia or reoxygenation in mouse and hamster RPTECs. ATPase activity decreased in mouse cells and remained stable in hamster cells under anoxia, whereas ATPase activity increased in the mouse and hamster cells during reoxygenation. Under normoxic conditions, the ATPase activity in the hamster cells was half of that in mouse cells. All experiments were performed nine times. ${ }^{~} \mathrm{P}<0.05$ vs. ctrl; ${ }^{*} \mathrm{P}<0.05$ vs. Anox; ${ }^{+} \mathrm{P}<0.05$, between ATPase activity of mouse and hamster RPTECs under normoxic conditions. Error bars correspond to the mean \pm standard deviation. RPTECs, renal proximal tubular epithelial cells; Mo, mouse; Hm, hamster; Anox, anoxia; ctrl, control culture under normoxia; Re-ox, reoxygenation.

In the mouse RPTECs, the level of p-4E-BP1 decreased by $53 \%$ following anoxia and increased following reoxygenation, but remained $21 \%$ lower than the level in normoxia. In the hamster RPTECs, the level of p-4E-BP1 decreased by $46 \%$ following anoxia but increased by $25 \%$ following reoxygenation (Fig. 3C and D).

ATPase activity decreases in mouse cells and remains stable in hamster cells under anoxia, whereas ATPase activity increases in mouse and hamster cells during reoxygenation. In the mouse RPTECs, ATPase activity decreased from $0.23 \pm 0.03 \mathrm{mU} / 100 \mu \mathrm{g}$ protein under normoxic conditions to $0.11 \pm 0.01 \mathrm{mU} / 100 \mu \mathrm{g}$ protein under anoxia, whereas it increased to $0.31 \pm 0.04 \mathrm{mU} / 100 \mu \mathrm{g}$ protein following reoxygenation. Compared with normoxia, the activity of ATPase in the hamster RPTECs did not alter during anoxia $(0.12 \pm 0.02$ vs. $0.12 \pm 0.01 \mathrm{mU} / 100 \mu \mathrm{g}$ protein), but increased to $0.21 \pm 0.02 \mathrm{mU} / 100 \mu \mathrm{g}$ protein following reoxygenation. Of note, under normoxic conditions, the activity of ATPase in the hamster cells was half of that observed in the mouse cells (Fig. 4).

Under normoxic conditions, hamster cells are less sensitive to ouabain-induced cell death than mouse cells. It has been noted in RPTECs that the majority of the activity of ATPase corresponds to $\mathrm{Na}^{+}-\mathrm{K}^{+}$-ATPase activity (12). Therefore, the observation that ATPase activity was $\sim 50 \%$ lower in hamster than mouse RPTECs under normoxic conditions may reflect the lower energy required for adequate $\mathrm{Na}^{+}-\mathrm{K}^{+}$-ATPase maintenance of a normal ion gradient in hamster cells. As the $\mathrm{Na}^{+}-\mathrm{K}^{+}$-ATPase pump preserves the $\mathrm{Na}^{+}$and $\mathrm{K}^{+}$ion gradient against passive leakage through ion channels, the lower energy demand of this pump may be the result of fewer ion channels in the hamster cell membrane. Once $\mathrm{Na}^{+}-\mathrm{K}^{+}$-ATPase pump activity is inhibited, deregulation of the intracellular ion concentration leads to cell death (17). The more ion channels there are, the faster the intracellular ion deregulation and cell death. Therefore, by inhibiting the $\mathrm{Na}^{+}-\mathrm{K}^{+}$-ATPase pump with a high concentration of ouabain and measuring the time span until cell death, the number or the functionality of ion channels can be assessed.

Cell imaging revealed that, under normoxic conditions, the ouabain-treated mouse RPTECs deteriorated significantly after $6 \mathrm{~h}$ and cell death occurred after $8 \mathrm{~h}$. The hamster RPTECs were less sensitive to ouabain as they declined considerably after $18 \mathrm{~h}$ and died after $24 \mathrm{~h}$ (Fig. 5A). The cell imaging results were confirmed biochemically. Following treatment for $4 \mathrm{~h}$ with ouabain, $88 \%$ of the hamster RPTECs survived, whereas only $57 \%$ of mouse RPTECs survived (Fig. 5B).

\section{Discussion}

Defining the mechanisms of resistance to I-R injury in mammalian hibernators may reveal novel therapeutic strategies against several human diseases, including coronary heart disease, cerebral ischemia, acute kidney injury and multiple organ failure (1-5).

Using RPTEC cultures, in consensus with previous studies $(8,9)$, the present study corroborated that, in contrast to the non-hibernator mouse, cells from the native hibernator Syrian hamster resisted cell death caused by warm anoxia or reoxygenation. One of the problems that mammalian hibernators have to cope with is cellular energy deprivation during periods of low tissue perfusion, as anaerobic glycolysis is less efficient than oxidative phosphorylation in ATP production. As expected, the experiments in the present study showed that cellular ATP content in the mouse RPTECs decreased during anoxia. By contrast, ATP remained stable in hamster RPTECs under anoxic conditions, indicating the existence of adaptive mechanisms which lower the energy requirements for cell survival. Cellular ATP content decreased in the RPTECs of both species following $2 \mathrm{~h}$ of reoxygenation, possibly as a result of the energetically demanding processes of cell repair following anoxia, including protein translation, correction of ion gradient disturbances and degradation of misfolded proteins. Confirming the above results, it was found that the alterations in the levels of activated AMPK were the inverse of changes in cellular ATP content, which is expected as AMPK is a sensor of cellular ATP and is activated in cases of low ATP levels (18).

In an attempt to clarify the mechanisms of ATP preservation in hamster RPTECs under anoxia, the present study evaluated the most energy demanding cellular processes. Protein translation is one such process (12). Two main factors that regulate protein translation are eIF $2 \alpha$ and 4E-BP1. Once eIF $2 \alpha$ is phosphorylated, it downregulates protein translation in general (19). In terms of 4E-BP1, it is phosphorylated by mammalian target of rapamycin complex 1 (mTORC1). Unphosphorylated 4E-BP1 inhibits protein translation (20). In hibernating squirrels, increased phosphorylation status of eIF $2 \alpha$ has been detected (21), whereas 4E-BP1 is hypophosphorylated (22). This sugestes that during low tissue perfusion mammalian hibernators can save energy by inhibiting global protein translation. 


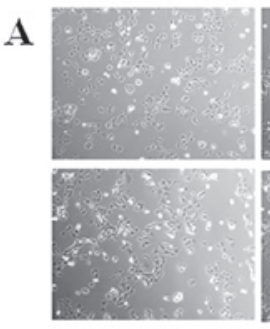

oh

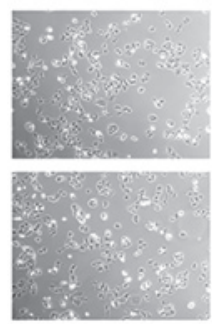

Oh

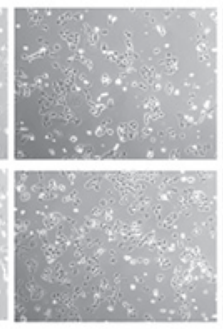

$2 \mathrm{~h}$

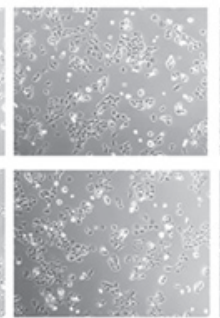

$4 h$

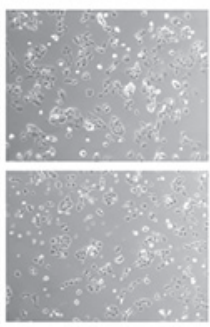

4h

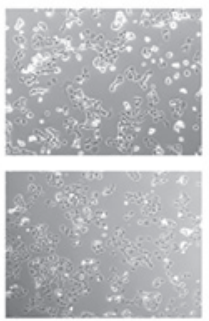

8 h

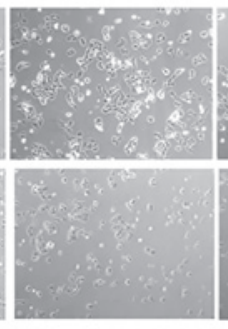

$6 \mathrm{~h}$

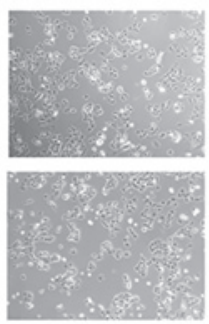

$12 \mathrm{~h}$

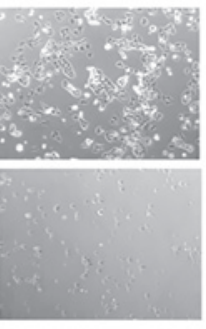

8h

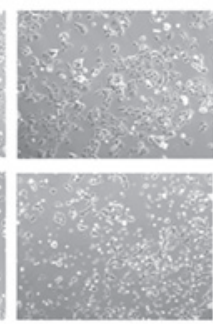

$18 \mathrm{~h}$

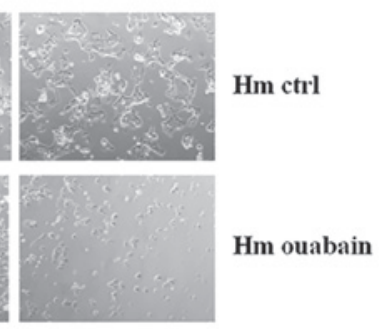

$24 h$

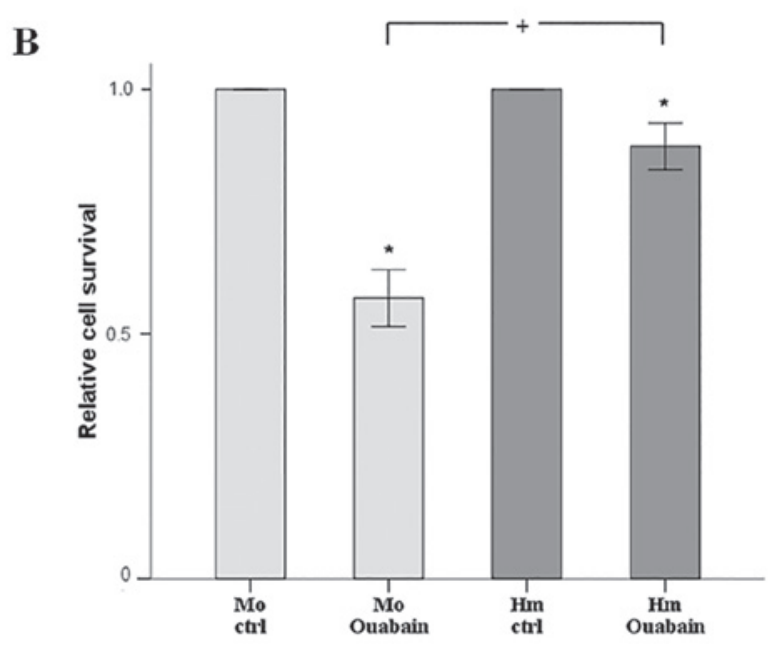

Figure 5. Sensitivity of mouse and hamster RPTECs to ouabain-induced cell death under normoxic conditions. (A) Cell imaging (magnification 100X) revealed that, under normoxic conditions, hamster cells survival was higher than mouse cell survival following treatment with a high concentration of $\mathrm{Na}^{+}-\mathrm{K}^{+}$-ATPase inhibitor ouabain. (B) Increased sensitivity of mouse cells to ouabain-induced cell death was confirmed biochemically using the XTT assay. All experiments were performed nine times. ${ }^{*} \mathrm{P}<0.05$ vs. ctrl; ${ }^{+} \mathrm{P}<0.05$ between cell survival of mouse and hamster RPTECs treated with ouabain. Error bars correspond to the mean \pm standard deviation. RPTECs, renal proximal tubular epithelial cells; Mo, mouse; Hm, hamster; Anox, anoxia; ctrl, control culture under normoxia; Re-ox, reoxygenation.

The experiments in the present study showed that warm anoxia increased p-eIF2 $\alpha$ and decreased p-4E-BP1 in mouse and hamster RPTECs. Therefore, protein translation was inhibited in cells from the two species, indicating that this adaptive mechanism is not specific for hibernators. In line with these results, other studies in cells from non-hibernators have also shown that, under hypoxia, eIF $2 \alpha$ is phosphorylated by endoplasmic reticulum-resident eIF2 kinase during the unfolded protein response (23), whereas mTORC1 is inhibited (24). When the cells were reoxygenated, the levels of p-eIF2a and p-4E-BP1 shifted towards the normoxic levels in mouse cells, but an overcorrection was observed in hamster cells. This may represent an adaptive mechanism for a faster recovery from anoxia-induced injury in hamster cells. Such an overcorrection has also been detected in p-4E-BP1 during interbout arousals in squirrels (22).

As protein synthesis decreased during anoxia in the RPTECs of the two species examined, the lower ATP content observed in anoxic mouse cells cannot be attributed solely to this. By contrast, the increased protein translation during reoxygenation in the mouse and hamster cells may contribute to the observed decrease in cellular ATP content. Therefore, in addition to protein translation, the present study examined the other most energy demanding cellular process, the function of the $\mathrm{Na}^{+}-\mathrm{K}^{+}$-ATPase pump. In the mammalian kidney, $\mathrm{Na}^{+}-\mathrm{K}^{+}$-ATPase consumes up to $40-70 \%$ of the produced ATP (12). Therefore, the majority of the ATPase activity measured in the present study corresponded to the function of the $\mathrm{Na}^{+}-\mathrm{K}^{+}$-ATPase pump. The experiments revealed that the activity of ATPase decreased during warm anoxia in the mouse RPTECs but remained stable in the hamster RPTECs. Under reoxygenation, the activity of ATPase was increased in cells from the two species, possibly in the context of energy consuming reparative processes. The latter contributes to the low cellular ATP content detected following $2 \mathrm{~h}$ of reoxygenation in the two cell types, despite the restoration of oxidative phosphorylation. 
Under normoxia, the activity of ATPase in the mouse RPTECs was 2-fold higher than that in the hamster RPTECs. This may reflect lower energy requirements for the function of $\mathrm{Na}^{+}-\mathrm{K}^{+}$-ATPase to be sufficient to preserve a normal ion gradient in hamster RPTECs. Therefore, it is likely that, under anoxic conditions, the net gain of ATP from the decreased protein synthesis in hamster RPTECs is sufficient for retaining a normal $\mathrm{Na}^{+}-\mathrm{K}^{+}$-ATPase function and cell survival. This agrees with the lower $\mathrm{Na}^{+}-\mathrm{K}^{+}$-ATPase activity observed in the renal cortex of active ground squirrels when compared with non-hibernating rabbits (25).

Regarding the curtailed energy demand for $\mathrm{Na}^{+}-\mathrm{K}^{+}$-ATPase function in hamster RPTECs, the channel arrest theory states that hibernators are less sensitive to anoxia, as they downregulate cell membrane $\mathrm{Na}^{+}$and $\mathrm{K}^{+}$ion channels under anoxia. Therefore, despite the decreased function of the $\mathrm{Na}^{+}-\mathrm{K}^{+}$-ATPase pump due to the reduced production of ATP, the decreased passive leakage of the $\mathrm{Na}^{+}$and $\mathrm{K}^{+}$ions allows the preservation of a normal ion gradient and cell survival under anoxia $(26,27)$. However, the experiments in the present study raise questions as to whether the predictions of the channel arrest theory are well-founded during normoxia. In such a case, the resistance of hamster RPTECs to anoxia pre-exists the encountering of anoxic conditions.

Once $\mathrm{Na}^{+}-\mathrm{K}^{+}$-ATPase activity is inhibited, deregulation of the intracellular ion concentration leads to cell death (17). The more ion channels there are, the faster the intracellular ion deregulation and cell death. In the present study, under normoxic conditions, more hamster cells survived than mouse cells following treatment with the $\mathrm{Na}^{+}-\mathrm{K}^{+}$-ATPase inhibitor ouabain, indicating that hamster RPTECs have fewer $\mathrm{Na}^{+}$and $\mathrm{K}^{+}$ion channels in the cell membrane than mouse RPTECs, and consequently less energy is necessary for adequate function of the $\mathrm{Na}^{+}-\mathrm{K}^{+}$-ATPase pump. Therefore, hamster RPTECs are prepaired for survival against an anoxic assault.

In conclusion, under anoxia, hamster RPTECs preserved energy homeostasis more efficiently than mouse RPTECs as a result of lower levels of ATP required for adequate $\mathrm{Na}^{+}-\mathrm{K}^{+}$-ATPase maintenance of a normal ion gradient; this is possibly due to fewer cell membrane $\mathrm{Na}^{+}$and $\mathrm{K}^{+}$ion channels. The increase in ATP from the depression of other energy-demanding processes, including protein synthesis, is sufficient for sustaining $\mathrm{Na}^{+}-\mathrm{K}^{+}$-ATPase function in hamster cells. The low energy required for $\mathrm{Na}^{+}-\mathrm{K}^{+}$-ATPase function and the faster recovery during reoxygenation contribute to the resistance of the native hibernator Syrian hamster RPTECs to anoxia or reoxygenation. Defining how mammalian hibernators cope with the repeated cycles of I-R may reveal possible molecular targets in humans for preventing warm I-R injury and potential novel therapeutic strategies against several I-R injury-induced human diseases.

\section{Acknowledgements}

Not applicable.

\section{Funding}

No funding was received.

\section{Availability of data and materials}

The analyzed datasets generated during the study are available from the corresponding author on reasonable request.

\section{Authors' contributions}

TE designed the study; GP and TE performed the experiments; TE, GP, GA, SG, VL and IS analyzed the results; TE wrote the manuscript with assistance from GP; IS supported all stages.

\section{Ethics approval and consent to participate}

Not applicable.

\section{Patient consent for publication}

Not applicable.

\section{Competing interests}

The authors declare that they have no competing interests.

\section{References}

1. Neri M, Riezzo I, Pascale N, Pomara C and Turillazzi E: Ischemia/reperfusion injury following acute myocardial infarction: A critical issue for clinicians and forensic pathologists. Mediators Inflamm 2017: 7018393, 2017.

2. Bakthavachalam P and Shanmugam PS: Mitochondrial dysfunction - Silent killer in cerebral ischemia. J Neurol Sci 375: 417-423, 2017.

3. Tsukamoto T, Chanthaphavong RS and Pape HC: Current theories on the pathophysiology of multiple organ failure after trauma. Injury 41: 21-26, 2010.

4. Bonventre JV and Yang L: Cellular pathophysiology of ischemic acute kidney injury. J Clin Invest 121: 4210-4221, 2011.

5. Lieberthal W and Nigam SK: Acute renal failure. I. Relative importance of proximal vs. distal tubular injury. Am J Physiol 275: F623-F631, 1998.

6. Carey HV, Andrews MT and Martin SL: Mammalian hibernation: Cellular and molecular responses to depressed metabolism and low temperature. Physiol Rev 83: 1153-1181, 2003.

7. Storey KB and Storey JM: Metabolic rate depression: The biochemistry of mammalian hibernation. Adv Clin Chem 52: 77-108, 2010.

8. Dave KR, Prado R, Raval AP, Drew KL and Perez-Pinzon MA: The arctic ground squirrel brain is resistant to injury from cardiac arrest during euthermia. Stroke 37: 1261-1265, 2006.

9. QuinonesQJ,ZhangZ,MaQ,SmithMP,SoderblomE,MoseleyMA, Bain J, Newgard CB, Muehlbauer MJ, Hirschey M, et al: Proteomic profiling reveals adaptive responses to surgical myocardial ischemia-reperfusion in hibernating arctic ground squirrels compared to rats. Anesthesiology 124: 1296-1310, 2016.

10. Levin E, Plotnik B, Amichai E, Braulke LJ, Landau S, Yom-Tov Y and Kronfeld-Schor N: Subtropical mouse-tailed bats use geothermally heated caves for winter hibernation. Proc Biol Sci 282: 20142781, 2015.

11. Dausmann KH, Glos J, Ganzhorn JU and Heldmaier G: Physiology: Hibernation in a tropical primate. Nature 429: 825-826, 2004.

12. Rolfe DF and Brown GC: Cellular energy utilization and molecular origin of standard metabolic rate in mammals. Physiol Rev 77: 731-758, 1997.

13. Venugopal $\mathrm{J}$ and Blanco G: On the many actions of Ouabain: Pro-cystogenic effects in autosomal dominant polycystic kidney disease. Molecules 22: 22, 2017.

14. Weinberg JM, Davis JA, Abarzua M, Smith RK and Kunkel R: Ouabain-induced lethal proximal tubule cell injury is prevented by glycine. Am J Physiol 258: F346-F355, 1990. 
15. Liu J, Periyasamy SM, Gunning W, Fedorova OV, Bagrov AY, Malhotra D, Xie Z and Shapiro JI: Effects of cardiac glycosides on sodium pump expression and function in LLC-PK1 and MDCK cells. Kidney Int 62: 2118-2125, 2002.

16. Cherniavsky-Lev M, Golani O, Karlish SJ and Garty H: Ouabain-induced internalization and lysosomal degradation of the $\mathrm{Na}^{+} / \mathrm{K}^{+}$-ATPase. J Biol Chem 289: 1049-1059, 2014.

17. Xiao AY, Wei L, Xia S, Rothman S and Yu SP: Ionic mechanism of ouabain-induced concurrent apoptosis and necrosis in individual cultured cortical neurons. J Neurosci 22: 1350-1362, 2002.

18. Mihaylova MM and Shaw RJ: The AMPK signalling pathway coordinates cell growth, autophagy and metabolism. Nat Cell Biol 13: 1016-1023, 2011.

19. Kilberg MS, Shan J and Su N: ATF4-dependent transcription mediates signaling of amino acid limitation. Trends Endocrinol Metab 20: 436-443, 2009.

20. Ma XM and Blenis J: Molecular mechanisms of mTOR-mediated translational control. Nat Rev Mol Cell Biol 10: 307-318, 2009.

21. Frerichs KU, Smith CB, Brenner M, DeGracia DJ, Krause GS Marrone L, Dever TE and Hallenbeck JM: Suppression of protein synthesis in brain during hibernation involves inhibition of protein initiation and elongation. Proc Natl Acad Sci USA 95: 14511-14516, 1998
22. van Breukelen F, Sonenberg N and Martin SL: Seasonal and statedependent changes of eIF4E and 4E-BP1 during mammalian hibernation: Implications for the control of translation during torpor. Am J Physiol Regul Integr Comp Physiol 287: R349-R353, 2004.

23. Koumenis C, Naczki C, Koritzinsky M, Rastani S, Diehl A, Sonenberg N, Koromilas A and Wouters BG: Regulation of protein synthesis by hypoxia via activation of the endoplasmic reticulum kinase PERK and phosphorylation of the translation initiation factor eIF2alpha. Mol Cell Biol 22: 7405-7416, 2002.

24. Arsham AM, Howell JJ and Simon MC: A novel hypoxiainducible factor-independent hypoxic response regulating mammalian target of rapamycin and its targets. J Biol Chem 278: 29655-29660, 2003.

25. Charnock JS and Simonson LP: Seasonal variations in the renal cortical $\left(\mathrm{Na}^{+}+\mathrm{K}^{+}\right)$-ATPase and $\mathrm{Mg}^{2+}$-ATPase of a hibernator, the ground squirrel (Spermophilus richardsonii). Comp Biochem Physiol B 60: 433-439, 1978.

26. Hochachka PW: Defense strategies against hypoxia and hypothermia. Science 231: 234-241, 1986.

27. Boutilier RG: Mechanisms of cell survival in hypoxia and hypothermia. J Exp Biol 204: 3171-3181, 2001. 